\title{
Caputo Standard $\alpha$-Family of Maps: Fractional Difference vs. Fractional
}

\author{
M. Edelman \\ Department of Physics, Stern College at Yeshiva University, \\ 245 Lexington Ave, New York, NY 10016, USA \\ Courant Institute of Mathematical Sciences, New York University, \\ 251 Mercer St., New York, NY 10012, USA
}

(Dated: November 12, 2018)

\begin{abstract}
In this paper the author compares behaviors of systems which can be described by fractional differential and fractional difference equations using the fractional and fractional difference Caputo Standard $\alpha$-Families of Maps as examples. The author shows that properties of fractional difference maps (systems with falling factorial-law memory) are similar to the properties of fractional maps (systems with power-law memory). The similarities (types of attractors, power-law convergence of trajectories, existence of cascade of bifurcations and intermittent cascade of bifurcations type trajectories, and dependence of properties on the memory parameter $\alpha$ ) and differences in properties of falling factorial- and power-law memory maps are investigated.
\end{abstract}


Unlike fractional calculus, whose history is more than three hundred years old, fractional difference calculus is relatively young - it is approximately thirty years old. This is probably the result of the fact that, despite the beautiful mathematics which arises during the development of fractional difference calculus, it doesn't have too many applications in nature and engineering. As it has been recently demonstrated, the simplest fractional difference equations (when a fractional difference on the left is equal to a nonlinear function on the right) are equivalent to maps with falling factorial-law memory. Falling factorial-law memory is asymptotically power-law memory with the rate of convergence proportional to the inverse of time (or number of iterations in discrete cases). It is difficult to distinguish power-law from asymptotically power-law memory which frequently appears in investigation of noisy natural systems. This is the major motivation for the presented work in which we study the simplest fractional difference equations with sine nonlinearity and compare their properties with properties of the corresponding systems with power-law memory.

\section{INTRODUCTION}

Systems with memory are common in biology, social sciences, physics, and engineering (see review [1]). Systems with power-law memory in many cases can be described by fractional differential equations [2 4]. If a natural system is a discrete one and can be described by a fractional difference equation, then the system's memory is falling factorial-law memory [5] 7], which is asymptotically power-law memory [8].

To study nonlinear systems with power-law memory Tarasov and Zaslavsky [9] introduced fractional maps, which are equivalent to the fractional differential equations of nonlinear systems experiencing periodic delta function-kicks. Fractional Riemann-Liouville and Caputo Standard Maps corresponding to the fractional differential equations with orders of derivatives $\alpha>1$ were used to investigate general properties of fractional dynamical systems in [9 15]. The notion of fractional $\alpha$-families of maps $(\alpha \mathrm{FM})$, which allowed the study of fractional Standard and Logistic Maps corresponding to $\alpha>0$, was introduced later in [1, 16, 17].

Fractional difference equations were investigated in many papers (see, e.g., [5, 7, 18, 22]). 
The authors of [8, 20 22] demonstrated that in some cases fractional difference equations are equivalent to maps with falling factorial-law memory (which we will call fractional difference maps), where falling factorial function is defined as

$$
t^{(\alpha)}=\frac{\Gamma(t+1)}{\Gamma(t+1-\alpha)} .
$$

Taking into account that falling factorial-law memory is asymptotically power-law memory (see Fig. 4 and Eq. (32) in this paper), we may expect that fractional difference maps have properties similar to the properties of fractional maps. Differences in the maps' properties due to the differences in the weights of the recent (with $(n-j) / n<<1$ ) states (a state is a set of variables which defines a system) at the time instants $t_{j}$ in the definition of the present state at time $t_{n}$ should be significant when $\alpha \in(0,1)$ (especially when $\alpha \rightarrow+0$ ), as it can be seen from Fig. 1 and comparison of Figs. 4 a and b.

The goal of the present paper is to conduct an investigation of fractional difference maps consistent with the previous research of fractional maps [1, 9 17] and make a step towards the understanding of the general properties of systems with asymptotically power-law memory. This will also lead to the understanding of the general properties of solutions of nonlinear fractional difference equations. In our investigation we use the fractional difference Caputo Standard $\alpha$-Family of Maps ( $\alpha$ FM) introduced in [8], which is an extension of the regular Standard Map [23 25]. A paper on the fractional difference Caputo Logistic $\alpha$ FM introduced in [8], which is an extension of the regular Logistic Map [26], will be the subject of a separate publication.

In the next section (Sec. II) we will recall the notions of fractional and fractional difference Caputo $\alpha$ FMs and in the following Sec. III we'll compare properties of the fractional and fractional difference Caputo Standard $\alpha$ FMs. 


\section{FRACTIONAL AND FRACTIONAL DIFFERENCE CAPUTO STANDARD $\alpha$ - FAMILIES OF MAPS}

\section{A. Fractional Caputo Standard $\alpha$-Family of Maps}

Fractional $\alpha$ FMs were introduced and investigated in [16, 17] (see also review [1]). They are identical to the following equation:

$$
\frac{d^{\alpha} x}{d t^{\alpha}}+G_{K}(x(t-\Delta)) \sum_{k=-\infty}^{\infty} \delta(t-(k+\varepsilon))=0
$$

where $\varepsilon>\Delta>0, \alpha \in \mathbb{R}, \alpha>0, \varepsilon \rightarrow 0$, with the initial conditions corresponding to the type of a fractional derivative to be used. $G_{K}(x)$ is a nonlinear function which depends on the nonlinearity parameter $K$.

The fractional Caputo Standard $\alpha \mathrm{FM}$ is generated by

- using in Eq. (2) the left-sided Caputo fractional derivative [2 4 ]

$$
\begin{aligned}
& { }_{0}^{C} D_{t}^{\alpha} x(t)={ }_{0} I_{t}^{N-\alpha} D_{t}^{N} x(t) \\
& =\frac{1}{\Gamma(N-\alpha)} \int_{0}^{t} \frac{D_{\tau}^{N} x(\tau) d \tau}{(t-\tau)^{\alpha-N+1}}, \quad(N=\lceil\alpha\rceil),
\end{aligned}
$$

where $N \in \mathbb{Z}, D_{t}^{N}=d^{N} / d t^{N},{ }_{0} I_{t}^{\alpha}$ is a fractional integral, $\Gamma()$ is the gamma function;

- using the initial conditions

$$
\left(D_{t}^{k} x\right)(0+)=b_{k}, \quad k=0, \ldots, N-1
$$

- and assuming

$$
G_{K}(x)=K \sin (x)
$$

Then, after the introduction $x^{(s)}(t)=D_{t}^{s} x(t)$, integration of Eq. (2) produces

$$
\begin{aligned}
& x_{n+1}^{(s)}=\sum_{k=0}^{N-s-1} \frac{x_{0}^{(k+s)}}{k !}(n+1)^{k} \\
& -\frac{K}{\Gamma(\alpha-s)} \sum_{k=0}^{n} \sin \left(x_{k}\right)(n-k+1)^{\alpha-s-1},
\end{aligned}
$$


where $s=0,1, \ldots, N-1$. We call the map Eq. (6) the fractional Caputo Standard $\alpha$ FM because in the $2 \mathrm{D}$ case $(\alpha=2)$ it can be reduced to the regular Standard Map (see [23]), which on a torus can be written as

$$
\begin{gathered}
p_{n+1}=p_{n}-K \sin \left(x_{n}\right), \quad(\bmod 2 \pi), \\
x_{n+1}=x_{n}+p_{n+1}, \quad(\bmod 2 \pi) .
\end{gathered}
$$

In [1, 16, 17] the Caputo Standard $\alpha \mathrm{FM}$ was investigated in detail for the case $\alpha \in[0,2]$ that is important in applications.

- For $\alpha=0$ the Caputo Standard $\alpha \mathrm{FM}$ is identically zero: $x_{n}=0$.

- For $0<\alpha<1$ the Caputo Standard $\alpha \mathrm{FM}$ is

$$
x_{n}=x_{0}-\frac{K}{\Gamma(\alpha)} \sum_{k=0}^{n-1} \frac{\sin \left(x_{k}\right)}{(n-k)^{1-\alpha}}, \quad(\bmod 2 \pi) .
$$

- For $\alpha=1$ the 1D Standard Map is the Circle Map with zero driving phase

$$
x_{n+1}=x_{n}-K \sin \left(x_{n}\right), \quad(\bmod 2 \pi) .
$$

- For $1<\alpha<2$ the Caputo Standard $\alpha \mathrm{FM}$ is

$$
\begin{aligned}
& p_{n+1}=p_{n}-\frac{K}{\Gamma(\alpha-1)}\left[\sum_{i=0}^{n-1} V_{\alpha}^{2}(n-i+1) \sin \left(x_{i}\right)\right. \\
& \left.+\sin \left(x_{n}\right)\right], \quad(\bmod 2 \pi), \\
& x_{n+1}=x_{n}+p_{0}-\frac{K}{\Gamma(\alpha)} \sum_{i=0}^{n} V_{\alpha}^{1}(n-i+1) \sin \left(x_{i}\right), \\
& (\bmod 2 \pi),
\end{aligned}
$$

where $V_{\alpha}^{k}(m)=m^{\alpha-k}-(m-1)^{\alpha-k}$.

- For $\alpha=2$ the Caputo Standard Map is the regular Standard Map as in Eqs. (7) and (8) above. 


\section{B. Fractional Difference Caputo Universal $\alpha$-Family of Maps}

As we mentioned in the Introduction, fractional difference calculus is a subject of extensive current research. To introduce the fractional difference Caputo Standard $\alpha$-Family of Maps we will use only one theorem (Theorem 3 from [8]):

Theorem 1 For $\alpha \in \mathbb{R}, \alpha \geq 0$ the Caputo-like difference equation

$$
{ }_{0}^{C} \Delta_{t}^{\alpha} x(t)=-G_{K}(x(t+\alpha-1)),
$$

where $t \in \mathbb{N}_{m}$, with the initial conditions

$$
\Delta^{k} x(0)=c_{k}, \quad k=0,1, \ldots, m-1, \quad m=\lceil\alpha\rceil
$$

is equivalent to the map with falling factorial-law memory

$$
\begin{aligned}
& x_{n+1}=\sum_{k=0}^{m-1} \frac{\Delta^{k} x(0)}{k !}(n+1)^{(k)} \\
& -\frac{1}{\Gamma(\alpha)} \sum_{s=0}^{n+1-m}(n-s-m+\alpha)^{(\alpha-1)} G_{K}\left(x_{s+m-1}\right),
\end{aligned}
$$

where $x_{k}=x(k)$, which we will call the fractional difference Caputo Universal $\alpha$-Family of Maps.

In this theorem ${ }_{0}^{C} \Delta_{t}^{\alpha}$ is defined by Anastassiou [19] for noninteger $\alpha>0$ fractional (left) Caputo difference operator as

$$
\begin{aligned}
& { }_{a}^{C} \Delta_{t}^{\alpha} x(t)={ }_{a} \Delta_{t}^{-(m-\alpha)} \Delta^{m} x(t) \\
& =\frac{1}{\Gamma(m-\alpha)} \sum_{s=a}^{t-(m-\alpha)}(t-s-1)^{(m-\alpha-1)} \Delta^{m} x(s),
\end{aligned}
$$

where $\Delta^{m}$ is the $m$-th power of the forward difference operator defined as $\Delta x(t)=x(t+$ $1)-x(t)$, extended in [8] to all real $\alpha \geq 0$ by defining ${ }_{a}^{C} \Delta_{t}^{m} x(t)=\Delta^{m} x(t)$ for $m \in \mathbb{N}_{0}$, where $\mathbb{N}_{t}=\{t, t+1, t+2, \ldots\}$.

The family of maps Eq. (15) is called universal because in the 2D case $(\alpha=2)$ after the introduction $p_{n}=\Delta x_{n-1}$ and with the assumption $G_{K}(x)=K G(x)$ it can be written as the regular Universal Map (see, e.g. [25])

$$
\begin{gathered}
p_{n+1}=p_{n}-K G\left(x_{n}\right), \\
x_{n+1}=x_{n}+p_{n+1} .
\end{gathered}
$$




\section{Integer-Dimensional Difference Universal Maps}

In the case of the integer $\alpha=m$ Eq. (13) can be written as

$$
\Delta^{m} x_{n}=-G_{K}\left(x_{n+m-1}\right),
$$

which for $m=0$ assumes the form

$$
x_{n+1}=-G_{K}\left(x_{n}\right)
$$

and for $m=1$ assumes the form

$$
x_{n+1}=x_{n}-G_{K}\left(x_{n}\right) .
$$

For $m>1$ lets define

$$
x_{n}^{0}=x_{n}, \quad x_{n}^{s}=\Delta x_{n-1}^{s-1}, \quad s=1,2, \ldots, m-1 .
$$

Then $x_{n}^{s}=\Delta^{s} x_{n-s}^{0}$ and Eq. 19 is equivalent to the $m$-dimensional map

$$
x_{n+1}^{s}=\sum_{k=s}^{m-1} x_{n}^{k}-G_{K}\left(x_{n}^{0}\right), \quad s=0,1, \ldots, m-1,
$$

which Jacobian $m \times m$ matrix $J_{\left(x_{n+1}^{0}, x_{n+1}^{1}, \ldots, x_{n+1}^{m-1}\right)}\left(x_{n}^{0}, x_{n}^{1}, \ldots, x_{n}^{m-1}\right)$ is

$$
\left|\begin{array}{cccccccc}
1-\dot{G}_{K}\left(x_{n}^{0}\right) & 1 & 1 & \ldots & 1 & \ldots & 1 & 1 \\
-\dot{G}_{K}\left(x_{n}^{0}\right) & 1 & 1 & \ldots & 1 & \ldots & 1 & 1 \\
-\dot{G}_{K}\left(x_{n}^{0}\right) & 0 & 1 & \ldots & 1 & \ldots & 1 & 1 \\
\ldots & \ldots & \ldots & \ldots & \ldots & \ldots & \ldots & \ldots \\
-\dot{G}_{K}\left(x_{n}^{0}\right) & 0 & 0 & \ldots & 0 & \ldots & 0 & 1
\end{array}\right| .
$$

The first column of this matrix can be written as the sum of the column with one in the first row and the remaining zeros and the column which is equal to $-\dot{G}_{K}(x)$ times the last column. Determinants of the corresponding matrices are 1 and 0 ; this is why the Jacobian determinant is equal to one and the map, similarly to the m-dimensional Universal Map (Eqs. (13) and (14) in [17]), is the m-dimensional volume preserving map. The mdimensional difference Universal and Universal Maps are identical only for the cases $m=1$ and $m=2$. 


\section{Fractional Difference Caputo Standard $\alpha$-Family of Maps}

For $G(x)=\sin (x)$ the map Eqs. (17) and (18) is equivalent to the regular Standard Map Eqs. (7) and (8). This is why we will call the map Eq. (15) with $G_{K}(x)=K \sin (x)$

$$
\begin{aligned}
& x_{n+1}=\sum_{k=0}^{m-1} \frac{\Delta^{k} x(0)}{k !}(n+1)^{(k)} \\
& -\frac{K}{\Gamma(\alpha)} \sum_{s=0}^{n+1-m}(n-s-m+\alpha)^{(\alpha-1)} \sin \left(x_{s+m-1}\right)
\end{aligned}
$$

the fractional difference Caputo Standard $\alpha \mathrm{FM}$.

- In the case $\alpha=0$ the 0D Standard Map turns into the Sine Map (see, e.g., [27])

$$
x_{n+1}=-K \sin \left(x_{n}\right), \quad(\bmod 2 \pi) .
$$

- For $0<\alpha<1$ the fractional difference Caputo Standard $\alpha \mathrm{FM}$ is

$$
\begin{aligned}
& x_{n+1}=x_{0} \\
& -\frac{K}{\Gamma(\alpha)} \sum_{s=0}^{n} \frac{\Gamma(n-s+\alpha)}{\Gamma(n-s+1)} \sin \left(x_{s}\right), \quad(\bmod 2 \pi),
\end{aligned}
$$

which after the $\pi$-shift of the independent variable $x \rightarrow x+\pi$ coincides with the "fractional sine map" proposed in [21].

- $\alpha=1$ difference Caputo Standard $\alpha \mathrm{FM}$ is identical to the Circle Map with zero driven phase Eq. [10]. The map considered in [21]

$$
x_{n+1}=x_{n}+K \sin \left(x_{n}\right), \quad(\bmod 2 \pi)
$$

is obtained from this map by the substitution $x \rightarrow x+\pi$.

- For $1<\alpha<2$ the fractional difference Caputo Standard $\alpha \mathrm{FM}$ is

$$
\begin{aligned}
& x_{n+1}=x_{0}+\Delta x_{0}(n+1)-\frac{K}{\Gamma(\alpha)} \\
& \times \sum_{s=0}^{n-1} \frac{\Gamma(n-s+\alpha-1)}{\Gamma(n-s)} \sin \left(x_{s+1}\right), \quad(\bmod 2 \pi),
\end{aligned}
$$


which after the introduction of $p_{n}=\Delta x_{n-1}$ can be written as a 2D map with memory

$$
\begin{aligned}
& p_{n}=p_{1}-\frac{K}{\Gamma(\alpha-1)} \\
& \times \sum_{s=2}^{n} \frac{\Gamma(n-s+\alpha-1)}{\Gamma(n-s+1)} \sin \left(x_{s-1}\right), \quad(\bmod 2 \pi), \\
& x_{n}=x_{n-1}+p_{n}, \quad(\bmod 2 \pi), \quad n \geq 1,
\end{aligned}
$$

which in the case $x_{0}=0$ is identical to the "fractional standard map" introduced in 21] (Eq. (18) with $\nu=\alpha-1$ there).

- The $\alpha=2$ difference Caputo Standard $\alpha$ FM is the regular Standard Map Eqs. (7) and (8).

\section{PROPERTIES OF THE FRACTIONAL AND FRACTIONAL DIFFERENCE CAPUTO STANDARD $\alpha$ FM}

The main properties of the Fractional Difference Caputo Standard $\alpha \mathrm{FM}$ and their differences from the properties of the Fractional Caputo Standard $\alpha \mathrm{FM}$ for $\alpha \in(0,2)$ are summarized in $\alpha-K_{c}$ diagram Fig. 1.

\section{A. Integer $\alpha$}

1. The Sine $\operatorname{Map}(\alpha=0)$

The bifurcation diagram for the case $\alpha=0$, the Sine Map Eq. (25), with $|K| \leq 2 \pi$ can be found in [27] and with $K \in[0.6,3.3]$ in Fig. 2a. It is easy to show by means of the standard stability analysis that the fixed point $x=0$ is a sink for $|K|<1$ and the period two $(T=2)$ point $x_{n+1}=-x_{n}$ is a sink for $1<K<2.262$ (at $K=2.262$ we have $\tan 2.029=-2.029$ and $\left|x_{n}\right|=2.029$ ). At $K=2.262$ two new $T=2$ sinks appear, which later (for larger $K$ ) bifurcate and give birth to the $T=4 \operatorname{sink}$ and so on. This period doubling cascade of bifurcations process leads to the onset of chaos at $K \approx 2.72$. In Fig. 1 the curves $K_{c 1 d}, K_{c 2 d}$, and $K_{c 3 d}$ intersect the line $\alpha=0$ at the points $1,2.262$, and 2.72 correspondingly. 


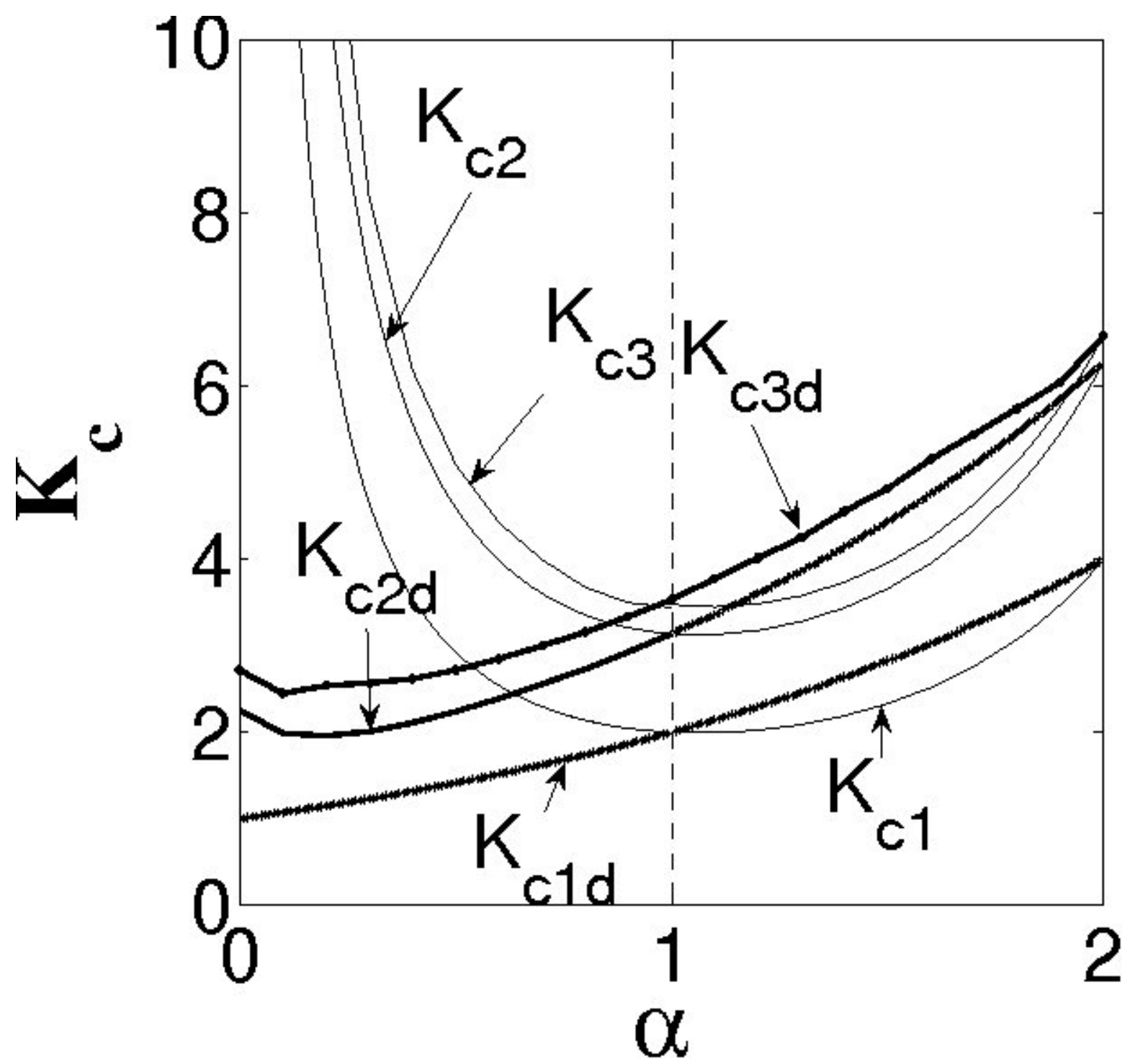

FIG. 1. $\alpha-K$ (bifurcation) diagrams for the Caputo (thin lines) and Fractional Difference Caputo (bold lines and extra index "d") Standard $\alpha$ FMs. The $(0,0)$ fixed point is stable in the area below the curve $K_{c 1}\left(K_{c 1 d}\right.$ for the difference map). The period two $(T=2)$ symmetric sink $\left(x_{n+1}=-x_{n}\right)$ is stable in the area between $K_{c 1}$ and $K_{c 2}\left(K_{c 1 d}\right.$ and $K_{c 2 d}$ for the difference map). $K_{c 3}$ ( $K_{c 3 d}$ for the difference map) is the border with chaos (above this curve). Cascade of bifurcations type trajectories can be found in the area near this curve (below it).

\section{The Circle Map with Zero Driven Phase $(\alpha=1)$}

The Circle Map with zero driven phase Eq. (10), which can also be called the 1D Standard Map, is investigated in [16, 17] and for $1.5<K<3.8$ is presented in Fig. 2b. In Fig. 1 the intersections of the curves $K_{c 1 d}, K_{c 2 d}$, and $K_{c 3 d}$ with the line $\alpha=1$ take place at the points 

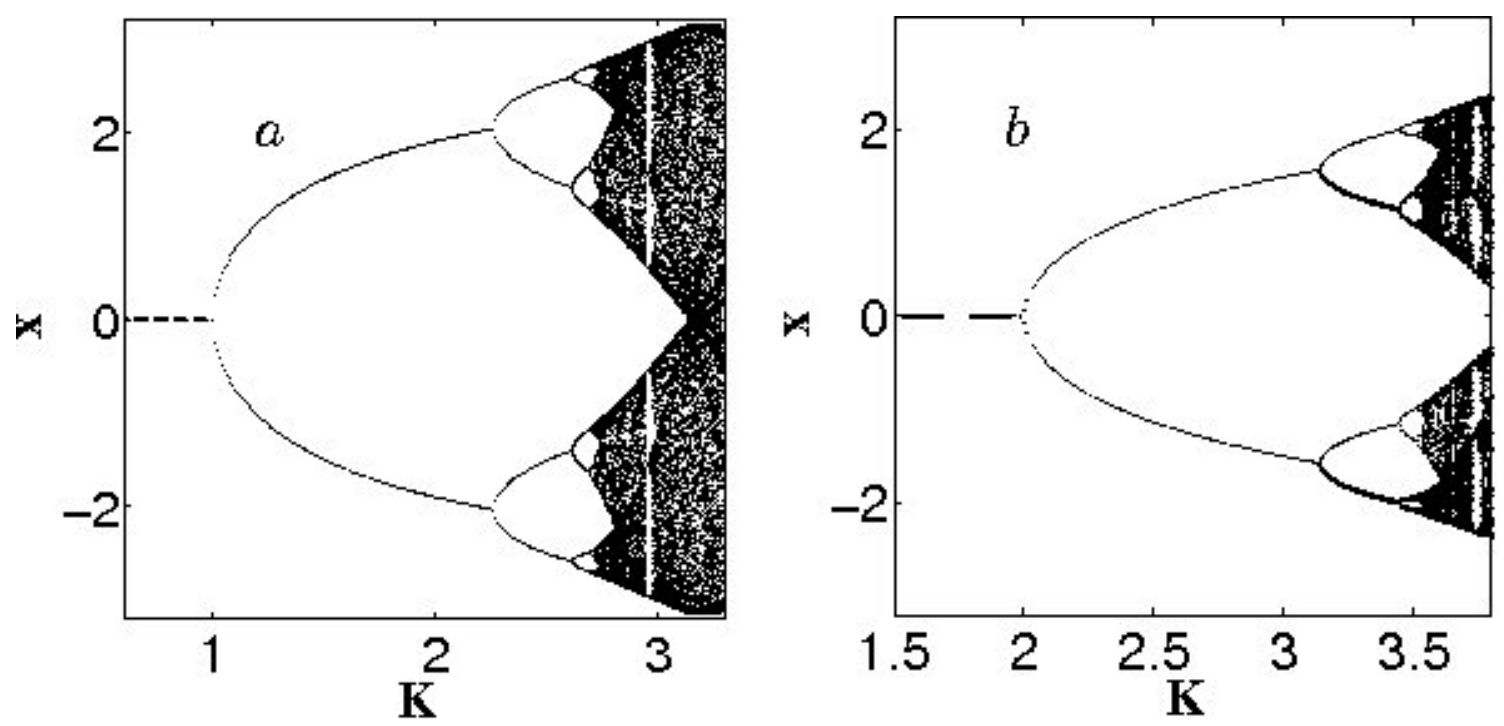

FIG. 2. Bifurcation diagrams for a). The Sine Map (difference map with $\alpha=0$ ) Eq. (25) and b). The Circle Map $(\alpha=1)$ with zero driven phase Eq. (10).

$2, \pi$, and 3.532 correspondingly (the same is true for the curves $K_{c 1}, K_{c 2}$, and $K_{c 3}$ ). Here we have to notice that the transition at $K=\pi$ is not from a $T=2 \operatorname{sink}$ to a $T=4 \operatorname{sink}$, but from the $x_{n+1}=-x_{n}$ period two sink to two $x_{n+1}=x_{n}+\pi$ period two sinks and in order to outline the whole bifurcation curve one should run computer codes with initial conditions $\pm x_{0}$ (something that the authors of [21] failed to notice). In Fig. 2b (and in Fig. 3) two sets of initial conditions correspond to two sets of points: the regular points $\left(x_{0}=0.1\right)$ and the bold points $\left(x_{0}=-0.1\right)$.

\section{The Standard Map $(\alpha=2)$}

The Standard Map (Chirikov Map) is one of the best-investigated maps (see [23, 24]). It demonstrates a universal generic behavior of the area-preserving maps whose phase space is divided into elliptic islands of stability and areas of chaotic motion. The $(0,0)$ elliptic point becomes unstable (elliptic-hyperbolic point transition) at $K=4$ and gives birth to two elliptic islands around the stable (for $4<K<2 \pi) T=2$ antisymmetric $\left(p_{n+1}=-p_{n}\right.$, $\left.x_{n+1}=-x_{n}\right)$ trajectory. At $K=2 \pi$ the antisymmetric $T=2$ point turns into two stable $T=2$ points with $p_{n+1}=-p_{n},\left|x_{n+1}-x_{n}\right|=\pi$. The following period doubling cascade of bifurcations leads to the disappearance of the islands of stability in the chaotic sea at $K \approx 6.6344$. In Fig. 1 the intersections of the curves $K_{c 1 d}, K_{c 2 d}$, and $K_{c 3 d}$ with the line 
$\alpha=2$ take place at the points $4,2 \pi$, and 6.6344 correspondingly (the same is true for the curves $K_{c 1}, K_{c 2}$, and $\left.K_{c 3}\right)$.

\section{B. $0<\alpha<1$}

Sample bifurcation diagrams for the fractional and fractional difference Caputo Standard $\alpha$ FM with $0<\alpha<1$ are presented in Fig. 3. One obvious difference between two $\alpha$ FMs is that as $\alpha$ decreases towards zero, bifurcation diagrams of the fractional difference maps Figs. 3 a, c, and e contract along the $K$-axis approaching the bifurcation diagram of the Sine Map Fig. 2 a, while the bifurcation diagrams of the fractional maps Figs. 3 b, d, and $\mathrm{f}$ expand along the $K$-axis.

The complete analysis of these bifurcation diagrams is not a subject of the present paper, but we'll outline some analytic results which were confirmed by the direct simulations of fractional maps.

Both maps, Eq. (9) and Eq. (26), can be written in the form

$$
x_{n}=x_{0}-\frac{K}{\Gamma(\alpha)} \sum_{k=0}^{n-1} W_{\alpha}(n-k) \sin \left(x_{k}\right),
$$

where $W_{\alpha}(s)=s^{\alpha-1}$ for the fractional map and $W_{\alpha}(s)=\Gamma(s+\alpha-1) / \Gamma(s)$ for the fractional difference map. Asymptotically, both expressions coincide (see Fig. 4) because

$$
\lim _{s \rightarrow \infty} \frac{\Gamma(s+\alpha)}{\Gamma(s+1) s^{\alpha-1}}=1, \quad \alpha \in \mathbb{R} .
$$

In the Sine Map and in the Circle Map with zero driven phase at the point where the $x=0$ sink becomes unstable it gives birth to a symmetric $T=2$ point in which $x_{n+1}=-x_{n}$. Following the results of our numeric simulations, let's assume that this property persists (asymptotically) for $\alpha \in(0,1)$. Eq. (31) can be written as

$$
\begin{aligned}
& x_{n+1}=x_{n}-\frac{K}{\Gamma(\alpha)}\left\{W_{\alpha}(1) \sin \left(x_{n}\right)\right. \\
& \left.+\sum_{k=0}^{n-1} \sin \left(x_{k}\right)\left[W_{\alpha}(n-k+1)-W_{\alpha}(n-k)\right]\right\} .
\end{aligned}
$$

Taking into account that $W_{\alpha}(n-k+1)-W_{\alpha}(n-k) \rightarrow 0$ as $n \rightarrow \infty$, after substitution 

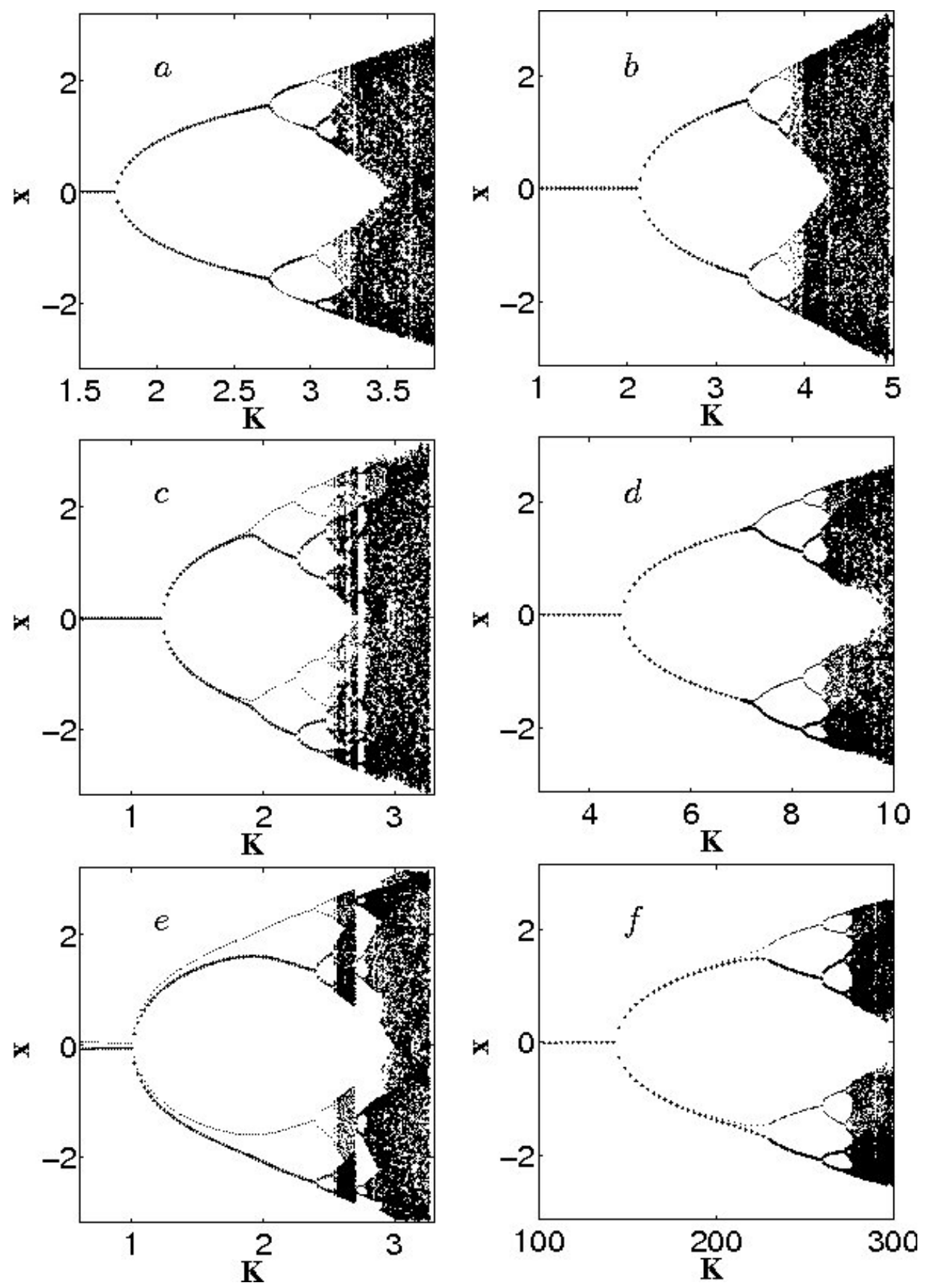

FIG. 3. Bifurcation diagrams for the fractional difference Caputo Standard $\alpha$ FM Eq. (26) ( $a$, $c$, and $e$ ) and the fractional Caputo Standard $\alpha$ FM Eq. (9) $(b, d$, and $f)$. The diagrams were obtained after 5000 iterations with the initial condition $x_{0}=0.1$ (regular points) and $x_{0}=-0.1$ (bold points). $\alpha=0.8$ in $a$ and $b ; \alpha=0.3$ in $c$ and $d ; \alpha=0.01$ in $e$ and $f$. 

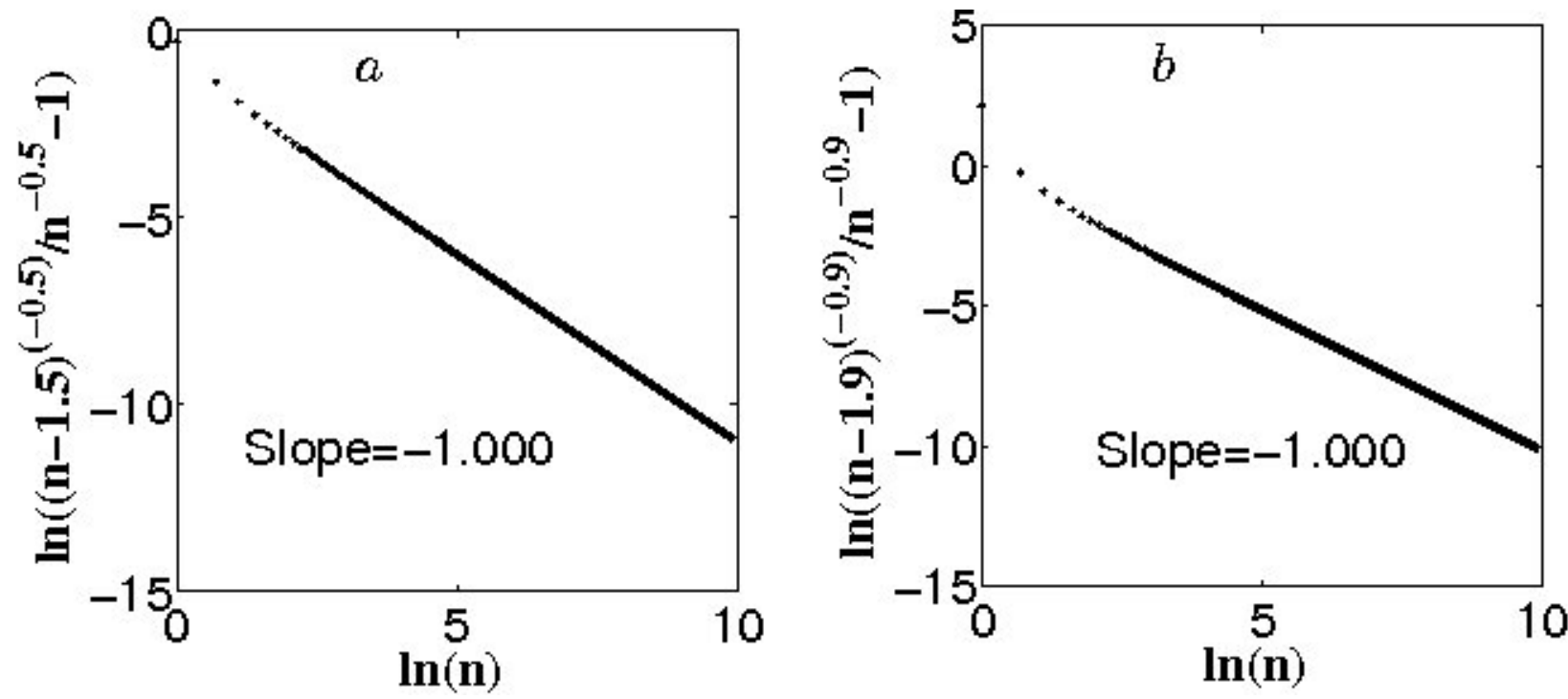

FIG. 4. Falling factorial $(n+\alpha-2)^{(\alpha-1)}$ to power law $n^{\alpha-1}$ ratio. $\alpha=0.5$ in (a) and $\alpha=0.1$ in (b).

$j=n-k$ for large $n$ we may write

$$
\begin{aligned}
& x_{n}=\frac{K}{2 \Gamma(\alpha)}\left\{W_{\alpha}(1)\right. \\
& \left.+\sum_{j=1}^{\infty}(-1)^{j}\left[W_{\alpha}(j+1)-W_{\alpha}(j)\right]\right\} \sin \left(x_{n}\right),
\end{aligned}
$$

where the alternating series on the right side converges because its terms converge to 0 monotonically. This equation has real non-trivial solutions when

$$
\begin{aligned}
& K>K_{c r 1} \\
& =\frac{2 \Gamma(\alpha)}{W_{\alpha}(1)+\sum_{j=1}^{\infty}(-1)^{j}\left[W_{\alpha}(j+1)-W_{\alpha}(j)\right]} .
\end{aligned}
$$

Numeric calculations of Eq. (35) with the corresponding functions $W_{\alpha}$ were performed to obtain the curves $K_{c 1}$ and $K_{c 1 d}$ for $\alpha \in(0,1)$ in Fig. 1 and they were also confirmed by the direct numeric simulations of the maps.

The direct numeric simulations of the maps show that for the fractional (this is not true for the fractional difference) Caputo Standard $\alpha \mathrm{FM}$, at the value of $K$ when the antisymmetric $T=2$ point becomes unstable, two new $T=2 \operatorname{sinks}$ appear with the property $\left|x_{n+1}-x_{n}\right|=\pi$. 
Then, an asymptotic consideration similar to the one presented above leads to

$$
\begin{aligned}
& \pm \pi=\frac{K}{\Gamma(\alpha)}\left\{W_{\alpha}(1)\right. \\
& \left.+\sum_{j=1}^{\infty}(-1)^{j}\left[W_{\alpha}(j+1)-W_{\alpha}(j)\right]\right\} \sin \left(x_{n}\right)
\end{aligned}
$$

and

$$
K>K_{c 2}=\pi K_{c 1} / 2
$$

The last equation was used to calculate the curve $K_{c 2}$ in Fig. 1. The curves $K_{c 2 d}, K_{c 3}$, and $K_{c 3 d}$ were obtained by the direct numeric simulations of the maps.

Periodic sinks $x=x_{l}$ (except the $x=0$ fixed point) exist only in the asymptotic sense. Trajectories starting at $x_{l}$ jump out of the sink and then converge asymptotically according to a power law $x-x_{l} \sim n^{-\alpha}$. This law of convergence to the $x=0$ sink is demonstrated in Fig. 5. For small $\alpha$ the rate of convergence is very slow. For the difference map even the rate of convergence itself is converging to its asymptotic value very slowly (Fig. 5.). Significance of the slow rate convergence for the explanation of the fact that in the fractional difference Caputo Standard $\alpha \mathrm{FM}$ with small values of $\alpha$ the bifurcation diagrams depend on the initial conditions (Fig. 6) is not investigated in the present paper. As can be seen from Fig. 6, the dependence of the bifurcation diagrams of the difference maps on the initial conditions is significant for $\alpha<0.2$. As in the case of fractional maps [1, 16, 17], individual trajectories of the fractional difference Standard $\alpha \mathrm{FM}$ with $0<\alpha<1$ in the area of the parameter values for which on the bifurcation diagram stable periodic $T>2$ sinks exist and the transition to chaos occurs are cascade of bifurcations type trajectories (CBTT) (see Fig. 7c). Even more complicated trajectories, including inverse cascades of bifurcations (Fig. $7 \mathrm{a}$ ) and trajectories with intermittent chaotic behavior (Fig. $7 \mathrm{~b}$ and $\mathrm{d}$ ), can be found in the fractional difference Standard $\alpha \mathrm{FM}$.

One of the consequences of the existence of CBTT is the dependence of bifurcation diagrams on the number of iterations after which they are calculated. In Fig. 8 some of the points which after 200 iterations are $T=2^{n}$ sinks, after 5000 iterations become $T=2^{n+1}$ sinks, and the corresponding bifurcation points shift to the left. 

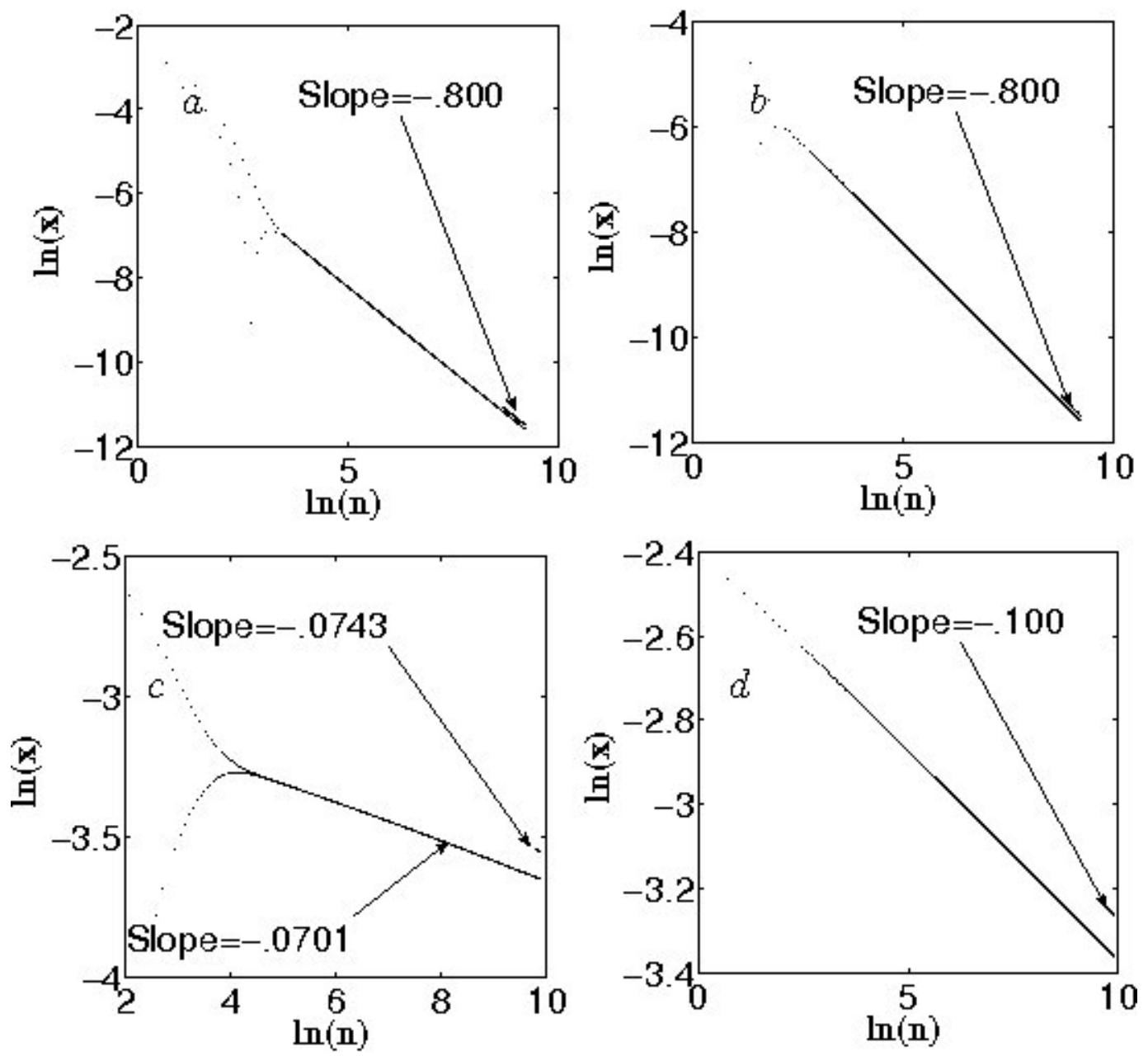

FIG. 5. Convergence of trajectories to the $x=0$ sink for the fractional ( $b$ and $d$ ) and fractional difference $\left(a\right.$ and $c$ ) Caputo Standard $\alpha \mathrm{FM}(\alpha \in(0,1))$. In all cases $x_{0}=0.1 .10000$ iterations, $\alpha=0.8$, and $K=1.5$ in $a$ and $b .20000$ iterations, $\alpha=0.1$, and $K=1.0$ in $c$ and $d$.

\section{C. $1<\alpha<2$}

In this section we'll apply the methods by which the evolution of the $(0,0)$ fixed point with the increase in $K$ was investigated for the fractional Standard Map with $1<\alpha<2$ in [1, 10, 14-17] to investigate the fractional difference Caputo Standard $\alpha$ FM for $1<\alpha<2$. As in the fractional Standard Map, when the $(0,0)$ sink becomes unstable it gives birth to the $T=2$ antisymmetric sink $x_{n+1}=-x_{n}, p_{n+1}=-p_{n}$, which later, at $K$ for which $x_{n}=\pi / 2$, turns into two $\pi$-shift $T=2 \operatorname{sinks}($ see Fig 9).

Assuming the existence of the antisymmetric $T=2 \operatorname{sink} x_{n+1}=-x_{n}, p_{n+1}=-p_{n}$ and 

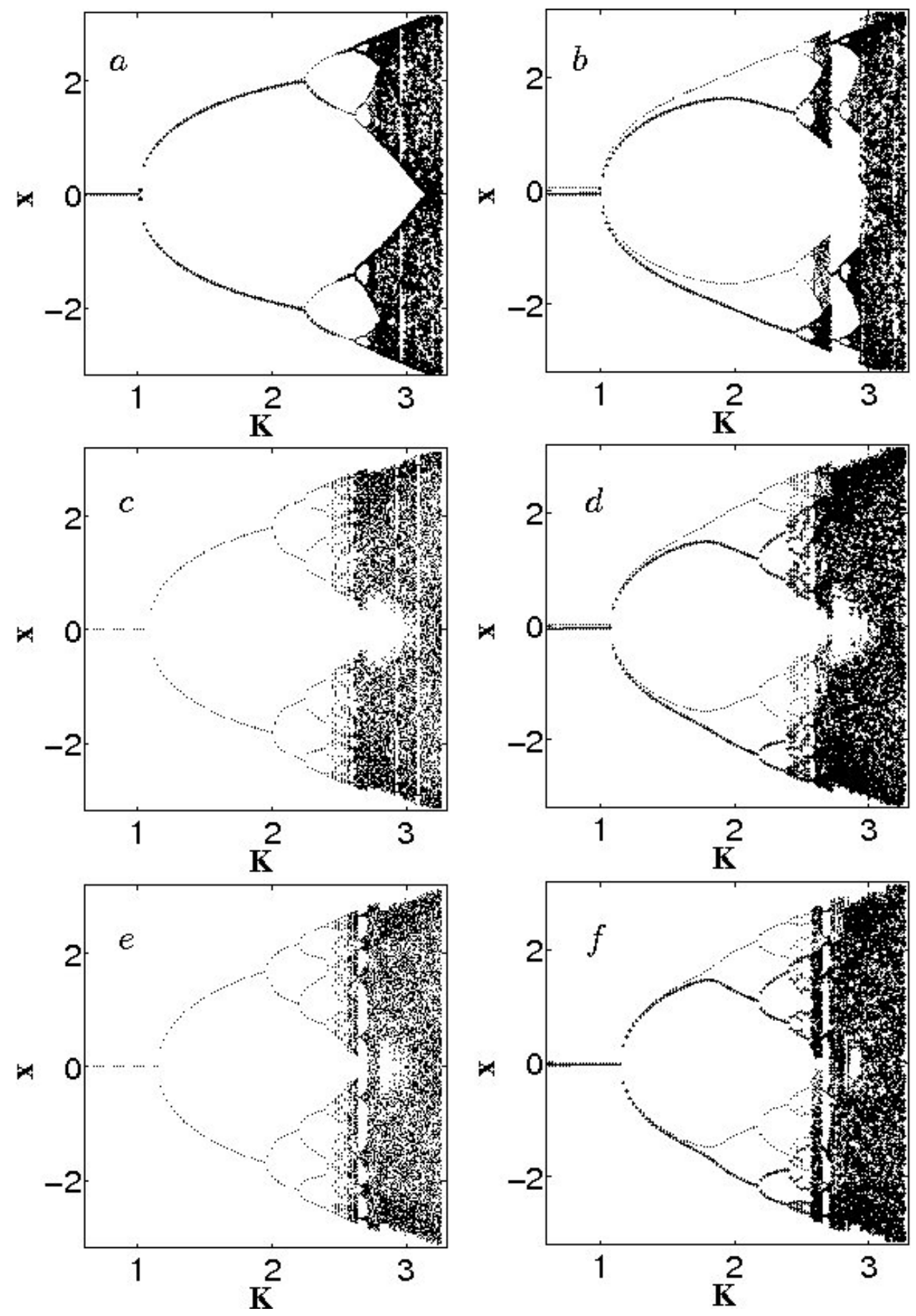

FIG. 6. Dependence of the fractional difference Caputo Standard $\alpha$ FM's bifurcation diagrams on the initial conditions for small $\alpha . \alpha=10^{-10}$ in $a$ and $b ; \alpha=.1$ in $c$ and $d ; \alpha=.2$ in $e$ and $f$. In $a$ and $b$ the bifurcation diagrams obtained after 200 iterations for each $K$. In $c, d, e$, and $f$ the bifurcation diagrams obtained after 5000 iterations for each $K$. The initial conditions: $x_{0}= \pm 0.001$ in $a ; x_{0}= \pm 0.00001$ in $c$ and $e ; x_{0}= \pm 0.1$ in $b, d$, and $f$. 

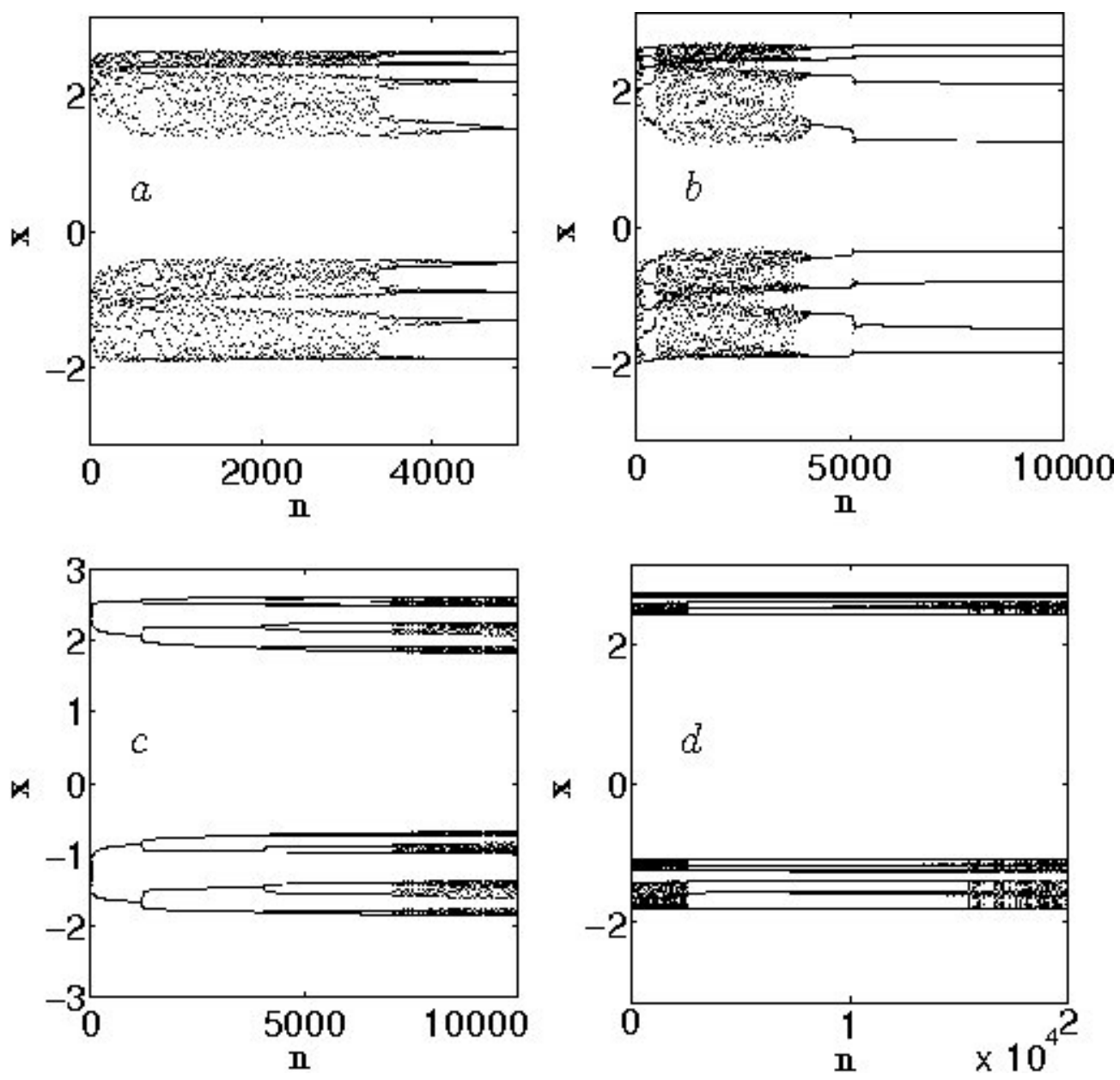

FIG. 7. Bifurcating trajectories in the fractional difference Caputo Standard $\alpha$ FM. Each figure represents a single trajectory with: a). $\alpha=0.2, K=2.52$, and $\left.x_{0}=0.1 ; \mathrm{b}\right) . \alpha=0.2, K=2.55$, and $\left.x_{0}=0.1 ; \mathrm{c}\right) . \alpha=0.1, K=2.41$, and $\left.x_{0}=0.1 ; \mathrm{d}\right) . \alpha=0.001, K=2.72$, and $x_{0}=0.003$;

following the same steps as in Sec. III B it is easy to derive from Eq. (29) for large $n$

$$
\begin{aligned}
& p_{n}=\frac{K}{2 \Gamma(\alpha-1)}\left\{W_{\alpha-1}(1)\right. \\
& \left.+\sum_{j=1}^{\infty}(-1)^{j}\left[W_{\alpha-1}(j+1)-W_{\alpha-1}(j)\right]\right\} \sin \left(x_{n}\right),
\end{aligned}
$$

where, as in Eq (31), $W_{\alpha}(s)=\Gamma(s+\alpha-1) / \Gamma(s)$. Eq. 30 for large $n$ gives $p_{n}=2 x_{n}$. 


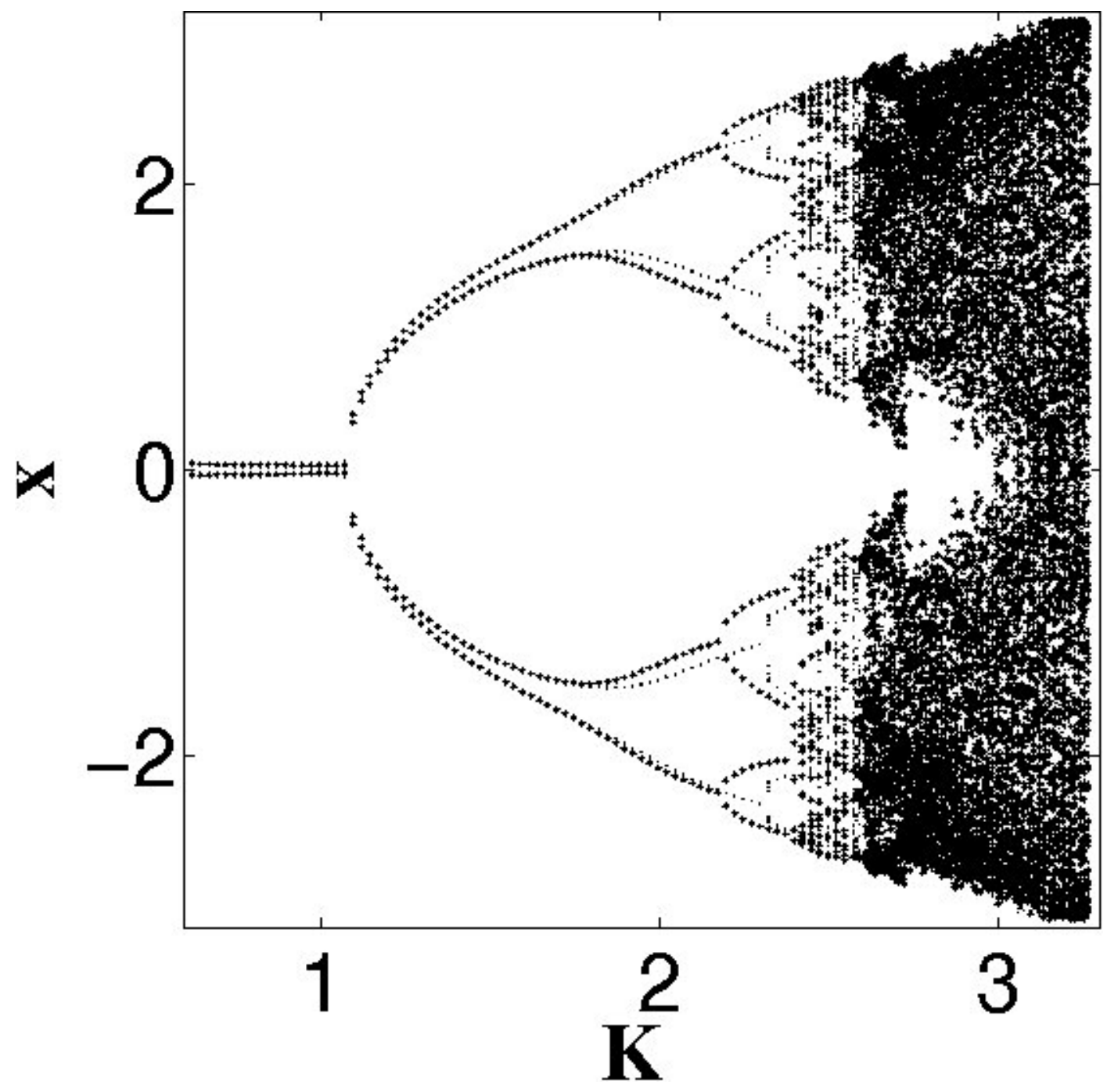

FIG. 8. Two bifurcation diagrams for the fractional difference Caputo Standard $\alpha$ FM with $\alpha=0.1$ and $x_{0}=0.1$ calculated after 200 iterations (regular points) and 5000 iterations (bold points).

Then, the equations defining the sink $\left(x_{n}, p_{n}\right)$ are

$$
\begin{aligned}
& x_{n}=\frac{K}{4 \Gamma(\alpha-1)}\left\{W_{\alpha-1}(1)\right. \\
& \left.+\sum_{j=1}^{\infty}(-1)^{j}\left[W_{\alpha-1}(j+1)-W_{\alpha-1}(j)\right]\right\} \sin \left(x_{n}\right), \\
& p_{n}=2 x_{n},
\end{aligned}
$$

from which follows that for $1<\alpha<2$

$$
K_{c 1 d}(\alpha)=2 K_{c 1 d}(\alpha-1)
$$

where $K_{c 1 d}(\alpha-1)$ is defined by Eq. (35). This result was confirmed by the direct numeric 

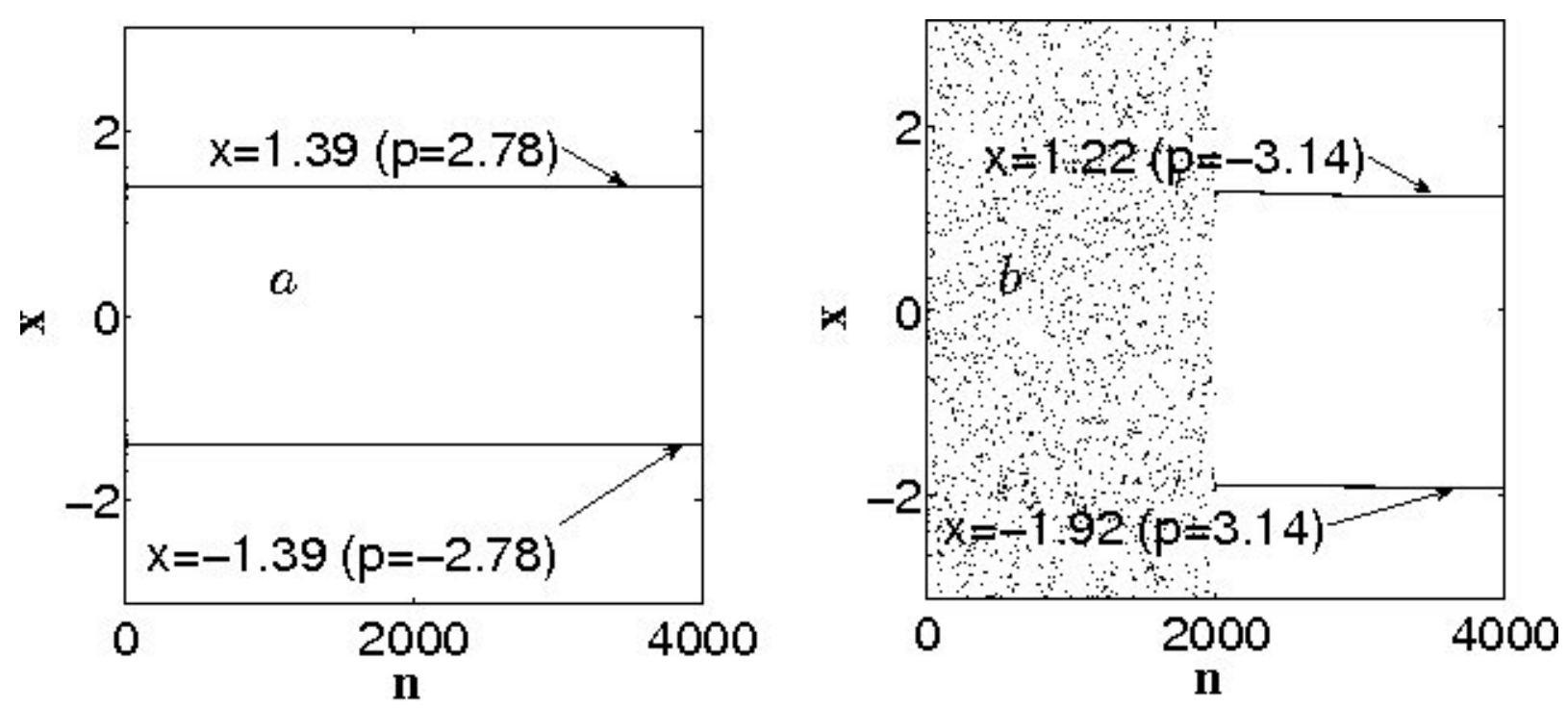

FIG. 9. Two $T=2$ trajectories for the fractional difference Caputo Standard $\alpha$ FM with $\alpha=1.5$ and $x_{0}=0$ and $\left.p_{0}=0.01: \mathrm{a}\right) . \mathrm{K}=4.0$ antisymmetric trajectory $\left.x_{n+1}=-x_{n}, p_{n+1}=-p_{n} ; \mathrm{b}\right)$. $\mathrm{K}=4.74 \pi$-shift trajectory with $\left|x_{n+1}-x_{n}\right|=\pi, p_{n+1}=-p_{n}$.

simulations of the maps and used to calculate the curve $K_{c 1 d}$ for $\alpha \in(1,2)$ in Fig. 1 .

In a similar way, assuming the existence of the antisymmetric $T=2 \operatorname{sink}\left|x_{n+1}-x_{n}\right|=\pi$, $p_{n+1}=-p_{n}$, asymptotically, the equations defining the sink $\left(x_{n}, p_{n}\right)$ can be written as

$$
\begin{aligned}
& \pm \pi=\frac{K}{2 \Gamma(\alpha-1)}\left\{W_{\alpha-1}(1)\right. \\
& \left.+\sum_{j=1}^{\infty}(-1)^{j}\left[W_{\alpha-1}(j+1)-W_{\alpha-1}(j)\right]\right\} \sin \left(x_{n}\right), \\
& p_{n}= \pm \pi .
\end{aligned}
$$

As for the fractional maps, for the fractional difference maps with $\alpha \in(1,2)$ the following holds

$$
K_{c 2 d}(\alpha)=\frac{\pi}{2} K_{c 1 d}(\alpha)
$$

The direct numeric simulations of the maps confirm this result. The $K_{c 3 d}$ curve is obtained by the direct map's numeric simulations.

As in the case of the fractional Caputo Standard Map, the trajectories in the fractional difference Caputo Standard Map converge to sinks according to a power law. But if in the case of the fractional Standard Map trajectories converge to the fixed point according to $x_{n} \sim n^{1-\alpha}$ and $p_{n} \sim n^{1-\alpha}$ (see, e.g. Fig. 1e in [15]), in the case of the fractional difference 

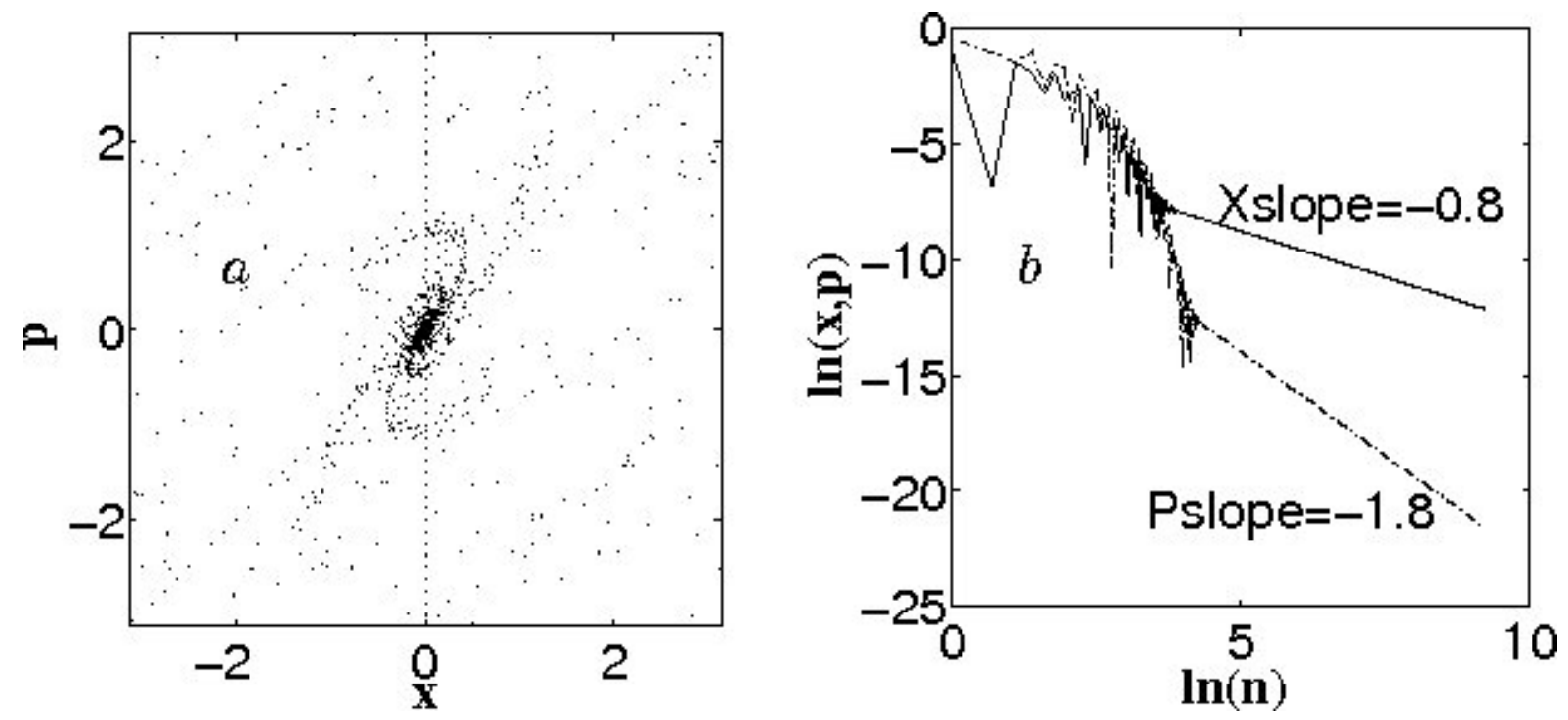

FIG. 10. The fractional difference Caputo Standard Map Eqs. (29) and (30) with $\alpha=1.8, K=2.5$ : a). Phase space obtained by performing 1000 iterations on each of the 50 trajectories with $x_{0}=0$ and $p_{1}=-3.1415+6.28 i / 50$, where $\left.0 \leq i<50 ; \mathrm{b}\right)$. Convergence to the $(0,0)$ sink of a trajectory with $x_{0}=0$ and $p_{0}=0.01$.

Standard Map the convergence is according to $x_{n} \sim n^{1-\alpha}$ and $p_{n} \sim n^{-\alpha}$ (see Fig. 10b). As we see, the rate of convergence of the $x$ variable is the same for both maps. The difference in the rates of convergence of the $p$ variable could be due to the difference in the definitions of momenta $p$ in two cases. The phase space of the "fractional Standard Map" (Eq. (18) from [21]) plotted for the same $\alpha=1.8$ and $K=2.5$ using 200 iterations on each of the 400 trajectories with $\left(x_{0}, p_{0}\right)=(-3.1415+6.28 i / 20,-3.1415+6.28 j / 20)$, where $\left.0 \leq i, j<20\right)$ is identical to the phase space of the fractional difference Standard Map Fig. 10a; the $\ln (x, p)$ vs. $\ln (n)$ graph for the "fractional Standard Map" with $\alpha=1.8, K=2.5, x_{0}=0.3$, and $p_{0}=0.1$ is also identical to the one in Fig. 10b. The phase portrait in Fig. 6 from [21] for $\alpha=1.8$ and $K=2.5$ with the structure of islands of stability and areas of chaotic motion is obviously incorrect.

As in the case of the fractional Standard Map [1, 10, 14 17], the most interesting features of the fractional difference Standard Map are CBTT and intermittent CBTT which appear below the border with chaos (curve $K_{c 3 d}$ in Fig. 1).

As in the fractional Caputo Standard Map, in the fractional difference Caputo Standard Map with $\alpha \in(1,2)$ intermittent CBTT can be found in $x$ vs. $n$ plots and reveal themselves 

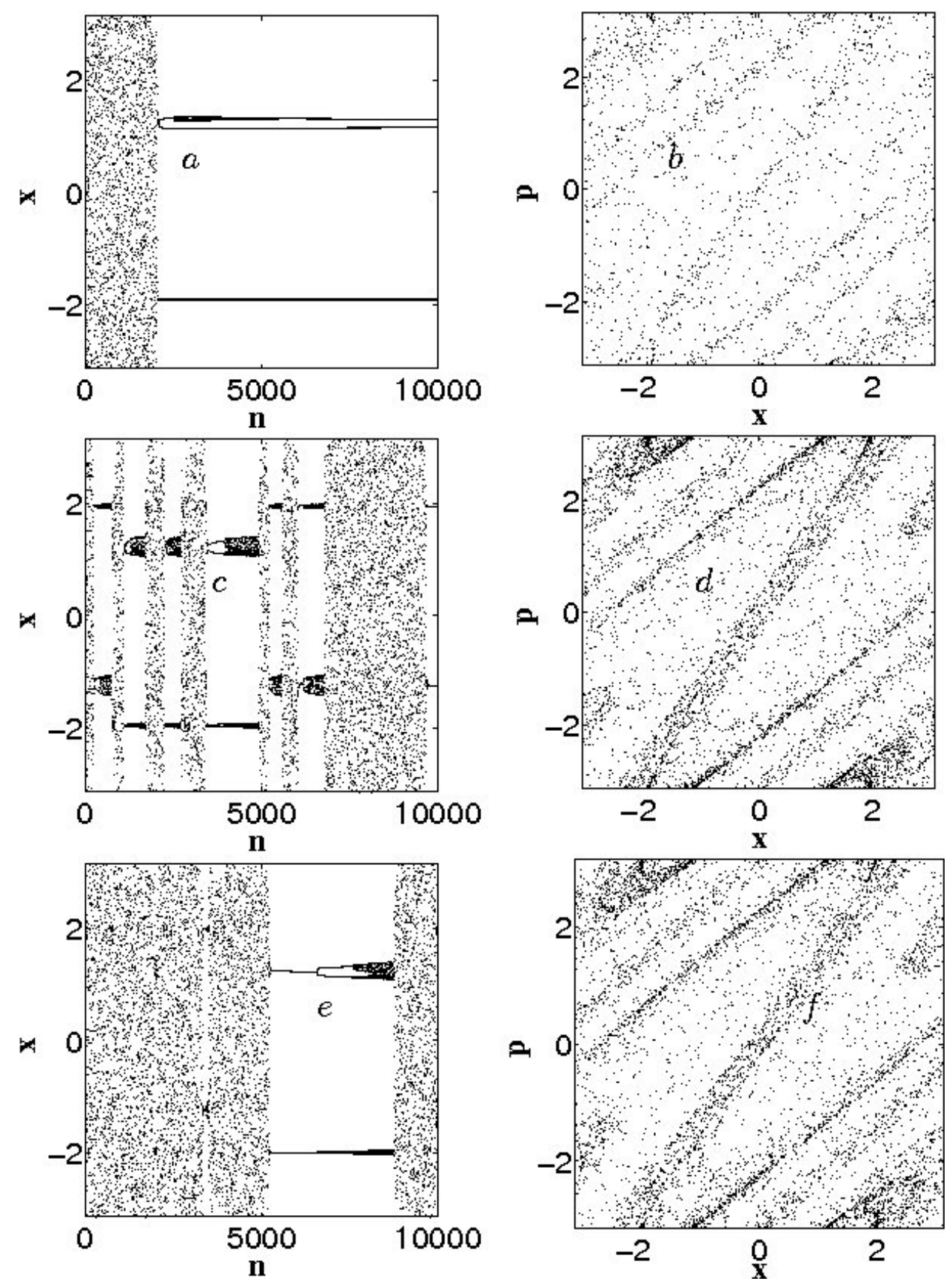

FIG. 11. Three single trajectories in the fractional difference Standard Map below the border with chaos in phase space $(b, d$, and $f)$ and in $x$ vs. $n$ graphs $(a, c$, and $e)$ with the initial conditions $x_{0}=0$ and $p_{0}=0.01 . \alpha=1.7$ and $K=5.43$ in $a$ and $b ; \alpha=1.5$ and $K=4.82$ in $c$ and $d ; \alpha=1.5$ and $K=4.92$ in $e$ and $f$.

best in the middle of the $(1,2)$ interval when $\alpha \approx 1.5$ (Fig. $11 a, c$, and $e$ ). In phase space intermittent CBTT are presented as dense dark areas embedded into chaotic attractors near 

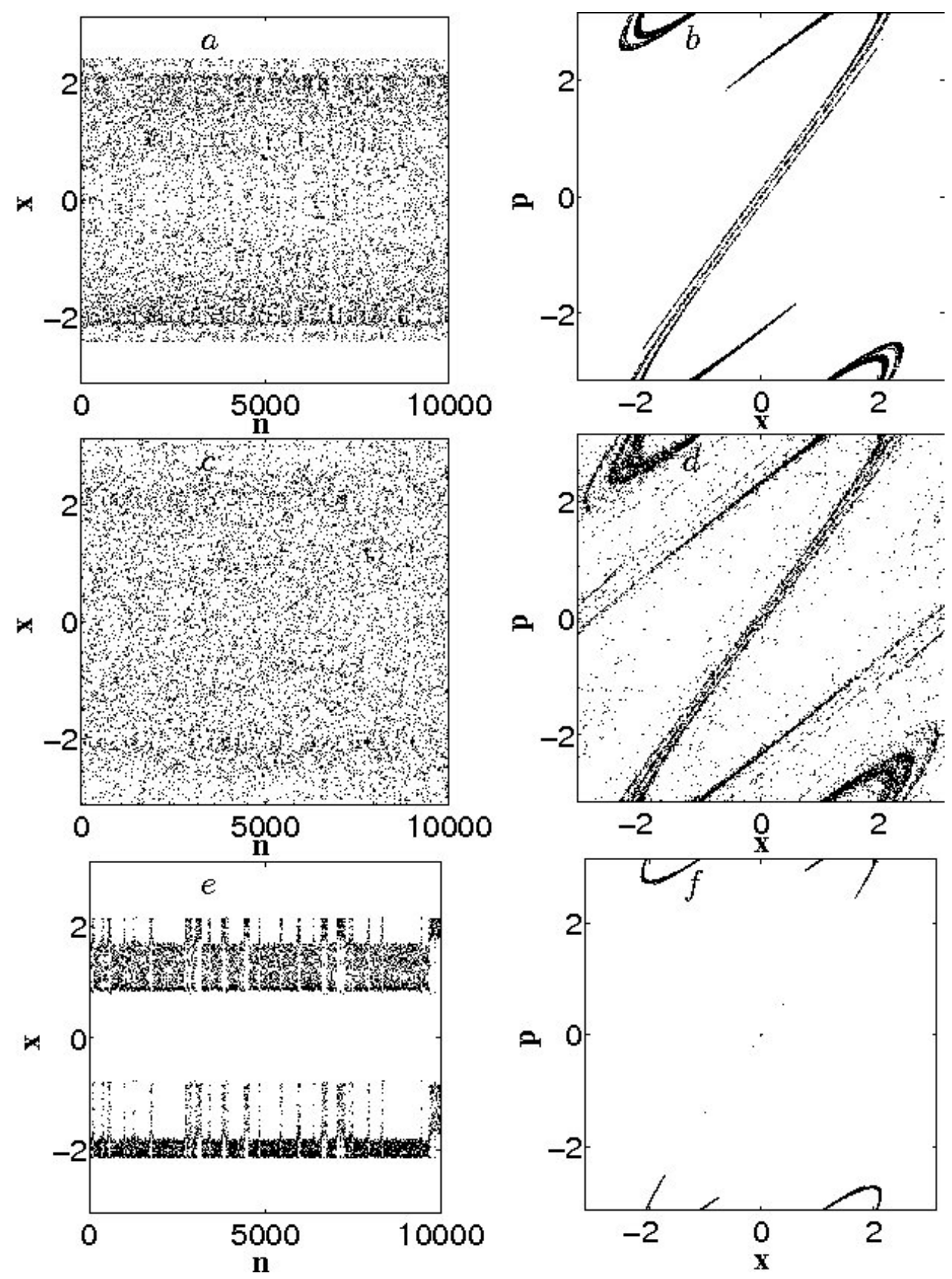

FIG. 12. Three single trajectories in the fractional difference Standard Map below the border with chaos in phase space $(b, d$, and $f)$ and in $x$ vs. $n$ graphs $(a, c$, and $e)$ with the initial conditions $x_{0}=0$ and $p_{0}=0.01 . \alpha=1.3$ and $K=4.3$ in $a$ and $b ; \alpha=1.3$ and $K=4.45$ in $c$ and $d ; \alpha=1.1$ and $K=3.8$ in $e$ and $f$.

points where $p= \pm \pi$ (Fig. $11 b, d$, and $f$ ). For small $\alpha$ (close to one) as $K$ increases towards the chaotic area periodic trajectories turn into chaotic attractors (Fig. 12). 


\section{CONCLUSION}

The main conclusion based on the results of the presented research is that systems with asymptotically power law-memory, similar to systems with power-law memory, demonstrate behaviors different from the behaviors of systems with no memory. The new properties include existence of attracting and intermittent cascade of bifurcations type trajectories, a common pattern in dependence of bifurcation diagrams on the memory parameter $\alpha$, and non-uniqueness of solutions (intersection of trajectories and overlapping of attractors) (see also [1, 15]).

The quanitative differences of properties of falling factorial-law memory maps from powerlaw memory maps are the results of the differences in weights of the recent states in the definition of the present state and are significant when $\alpha \rightarrow+0$. Behavior of systems with small values of $\alpha$ appears to be the most interesting (see Figs. 3 e, f, 6), 7, and 8 , It is interesting to notice that the case of small $\alpha$ plays an important role in biological applications (see, e.g., [1]). It has been shown recently [28, 29] that processing of external stimuli by individual neurons can be described by fractional differentiation. The orders of fractional derivatives $\alpha$ obtained for different types of neurons fall within the interval $[0,1]$. For neocortical pyramidal neurons it is quite small $\alpha \approx 0.15$. We suggest that it will be important for biological applications to conduct more theoretical research of the maps with small $\alpha$ and to make a comparison with experimental biological results.

\section{ACKNOWLEDGMENTS}

The author acknowledges support from the Joseph Alexander Foundation, Yeshiva University. The author expresses his gratitude to E. Hameiri, H. Weitzner, and G. Ben Arous for the opportunity to complete this work at the Courant Institute and to V. Donnelly for technical help.

[1] M. Edelman, in: Nonlinear Dynamics and Complexity, edited by A. Afraimovich, A. C. J. Luo, and X. Fu (Springer, New York, 2014), pp. 79-120. 
[2] S. G. Samko, A. A. Kilbas, and O. I. Marichev, Fractional Integrals and Derivatives Theory and Applications (Gordon and Breach, New York, 1993).

[3] A. A. Kilbas, H. M. Srivastava, and J. J. Trujillo, Theory and Application of Fractional Differential Equations (Elsevier, Amsterdam, 2006).

[4] I. Podlubny, Fractional Differential Equations (Academic Press, San Diego, 1999).

[5] H. L. Gray and N.-F. Zhang, Math. Comput. 50, 513 (1988).

[6] K. S. Miller and B. Ross, "Fractional Difference Calculus", in: Univalent Functions, Fractional Calculus, and Their Applications, edited by H. M. Srivastava and S. Owa (Ellis Howard, Chichester, 1989), pp. 139-151.

[7] F. M. Atici and P. W. Eloe, Proc. Am. Math. Soc. 137, 981 (2009).

[8] M. Edelman, Discontinuity, Nonlinearity, and Complexity (2014), (submitted); (see also arXiv:1404.4906 v3, 2014).

[9] V. E. Tarasov and G. M. Zaslavsky, J. Phys. A 41, 435101 (2008).

[10] M. Edelman and V. E. Tarasov, Phys. Lett. A 374, 279 (2009).

[11] V. E. Tarasov, J. Phys. A: Math. Theor. 42, 465102 (2009).

[12] V. E. Tarasov, J. Math. Phys. 50, 122703 (2009).

[13] V. E. Tarasov, Fractional Dynamics: Application of Fractional Calculus to Dynamics of Particles, Fields, and Media (Springer, HEP, New York, 2011).

[14] M. Edelman, Commun. Nonlin. Sci. Numer. Simul. 16, 4573 (2011).

[15] M. Edelman and L. A. Taieb, "New Types of Solutions of Non-Linear Fractional Differential Equations", in: Advances in Harmonic Analysis and Operator Theory; Series: Operator Theory: Advances and Applications, edited by A. Almeida, L. Castro, and F.-O. Speck (Basel, Springer, 2013) Vol. 229, pp. 139-155.

[16] M. Edelman, Discontinuity, Nonlinearity, and Complexity 1, 305 (2013).

[17] M. Edelman, Chaos 23, 033127 (2013).

[18] R. P. Agarwal, Difference equations and inequalities (Marcel Dekker, New York, 2000).

[19] G. A. Anastassiou, http://arxiv.org/abs/0911.3370 (2009).

[20] F. Chen, X. Luo, and Y. Zhou, Adv. Differ. Eq. 2011, 713201 (2011).

[21] G.-C. Wu, D. Baleanu, and S.-D. Zeng, Phys. Lett. A 378, 484 (2014).

[22] G.-C. Wu, D. Baleanu, Nonlin. Dyn. 75, 283 (2014).

[23] B. V. Chirikov, Phys. Rep. 52, 263 (1979) 
[24] A. J. Lichtenberg and M. A. Lieberman, Regular and Chaotic Dynamics (Springer, Berlin. 1992).

[25] G. M. Zaslavsky, Hamiltonian Chaos and Fractional Dynamics (Oxford University Press, Oxford, 2008).

[26] R. M. May, Nature 261, 459 (1976).

[27] C. C. Lalescu, arXiv:1011.6552 (2010).

[28] B. N. Lundstrom, M. H. Higgs, W. J. Spain, and A. L. Fairhall, Nature Neuroscience 11, 1335-1342 (2008).

[29] B. N. Lundstrom, A. L. Fairhall, and M. Maravall, The Journal of Neuroscience 30, 5071 (2010). 